International Applied Statistics in Medicine Conference, Dallas, TX; 1995. In press.

31. Benneyan JC. The importance of modeling discrete data in SPC. In: Proceedings of the 10th International Conference of the Israel Society for Quality, Israel; 1994:640-646.

32. Benneyan JC. Design of statistical $g$ control charts for nosocomial infection. In: Proceedings of the First International Applied Statistics in Medicine Conference; Dallas, TX; 1995. In press.

33. Marshall RJ. A review of methods for the statistical analysis of spatial patterns of disease. J R Stat Soc Assoc 1991;154 part 3:421-441.

34. Wartenberg D, Greenburg M. Characterizing cluster studies: a review of the literature. Presented at the Conference on Statistics and Computing in Disease Clustering; Vancouver, British Columbia, Canada; July 21-22, 1994.

35. Maskarinec G. Investigating increased incidence of events in the Islands: a Hawaii Department of Health perspective. Stat Med 1996. In press.

\title{
Simultaneous Influenza and Pneumococcal Vaccines
}

\section{by Gina Pugliese, RN, MS Medical News Editor}

A recent study concluded that simultaneous administration of pneumococcal and influenza vaccines to the elderly is safe and adverse reactions are mild. This is good news, because it may provide a strategy to increase the currently poor immunization rates for two infections that are major causes of morbidity and mortality among the elderly.

The coverage of influenza vaccination is approximately $40 \%$, and the coverage of pneumococcal vaccination is around $10 \%$. The Immunization Practices Advisory Committee (ACIP) recommends that both vaccines be administered to persons aged 65 years or older. It is clear that there needs to be methods to improve vaccine use, and one possible improvement would be simultaneous administration of the two vaccines.

Simultaneous administration of these vaccines has been shown not to interfere with the antibody response, and the possibility of giving both vaccines at the same time is included in the ACIP recommendation. However, there are few data regarding the effects of the two vaccines when given at the same time.

Dr. Pekka Honkanen and colleagues from the National Public Health Institute in Oulu, Finland, conducted a study of 9,336 persons in Northern Finland aged 65 years or older, of whom 4,581 persons received influenza vaccine and 4,755 persons received influenza and pneumococcal vaccines. No serious reactions were observed in any vaccine recipients. The incidence of local reactions was 284 per 1,000 vaccinations in the influenza-vaccinated group and 441 per 1,000 vaccinations in the influenza-pneumococcal-vaccinated group. The incidence of fever (at least $37.5^{\circ} \mathrm{C}$ ) was 10 and 24 per 1,000 , respectively. The frequency of local reactions decreased with advancing age.

The authors conclude that simultaneous administration of pneumococcal and influenza vaccine proved to be safe, and, when indicated, the vaccines should be given at the same time.

FROM: Honkanen PO, Keistinen T, Sirkka-Liisa K. Reactions following administration of influenza vaccine alone or with pneumococcal vaccine to the elderly. Arch Intern Med 1996;156:205-208. 